\title{
Correlation Between Hybrid Capture II High-Risk Human Papillomavirus DNA Test Chemiluminescence Intensity From Cervical Samples With
Follow-Up Histologic Results
}

A Cytologic/Histologic Review of 367 Cases

Stewart M. Knoepp, MD, PhD'; Diane L. Kuebler, MD²; and David C. Wilbur, MD²

BACKGROUND: The Hybrid Capture II high-risk human papillomavirus (hrHPV) DNA test is a US Food and Drug Administration-approved nucleic acid hybridization assay using chemiluminescence for the semiquantitative detection of hrHPV in cervical samples. Patient samples and controls are used to calculate results as negative for hrHPV if <1.0, positive for hrHPV if $>2.5$, and "equivocal" if between 1.0 and 2.5. METHODS: The authors reported on the cervical histologic results of 209 patients demonstrating "equivocal" results for hrHPV from SurePath (204 patients) or ThinPrep (5 patients) vials, and compared patients in this cohort with atypical squamous cells of undetermined significance (ASC-US) cytology on the index cervical Papanicolaou (Pap) test (Group 1; $\mathrm{n}=148$ patients) with a patient cohort demonstrating unequivocal positive hrHPV test results (Group 2; $\mathrm{n}=148$ patients). The chemiluminescence intensity of hrHPV tests from patients in Group 2 were correlated with the presence and severity of dysplasia on subsequent histologic results, and patients were thereby stratified for their subsequent risk of cervical intraepithelial neoplasia (CIN) types II/III. RESULTS: Approximately 97\% of hrHPV tests demonstrating "equivocal" results were found to be positive at the time of retesting, and $15 \%$ of biopsied cases demonstrated CIN II or III. Results of follow-up histology after an ASC-US diagnosis, expressed as a percentage of the biopsied cohort, were: CIN II/III: $16.5 \%$ in Group 1 and 22.4\% in Group 2; CIN I: $27 \%$ in Group 1 and 23.5\% in Group 2; and negative: $56.5 \%$ in Group 1 and $54.1 \%$ in Group 2. Chemiluminescence intensity did not appear to be correlated with the severity of dysplasia. CONCLUSIONS: The percentage of high-grade CIN in the "equivocal" hrHPV cohort is highly significant and therefore the management of these patients should be similar to the unequivocally positive population. After an unequivocal positive hrHPV test, the hrHPV chemiluminescence intensity does not appear to further predict the rate of high-grade CIN. Cancer (Cancer Cytopathol) 2010;118:209-17. (C) 2010 American Cancer Society.

KEY WORDS: cervical cytology, human papillomavirus (HPV) testing, SurePath, Hybrid Capture II.

Corresponding author: Stewart M. Knoepp, MD, PhD, Department of Pathology, University of Michigan, 2G332UH, 1500 East Medical Center Drive, SPC 5054, Ann Arbor, MI 48109; Fax: (734) 763-4095; sknoepp@umich.edu

'Department of Pathology, University of Michigan, Ann Arbor, Michigan; ${ }^{2}$ Department of Pathology, Massachusetts General Hospital, Harvard Medical School, Boston, Massachusetts

Received: February 12, 2010; Revised: April 13, 2010; Accepted: May 21, 2010

Published online June 24, 2010 in Wiley InterScience (www.interscience.wiley.com)

DOI: 10.1002/cncy.20093, www.interscience.wiley.com 
High-risk types of human papillomavirus (hrHPV) have been implicated in the vast majority of cases of high-grade cervical dysplasia and cervical carcinoma, ${ }^{1}$ and hrHPV testing has emerged as a highly sensitive test for the presence of cervical neoplasia. ${ }^{2}$ A wide variety of tests are currently available for the detection of hrHPV. Although the variety of available tests provides many options for laboratories using a hrHPV test, it is important that laboratories use analytically as well as clinically validated assays. ${ }^{3}$

Unlike low-risk types of HPV, in which the HPV genome is typically maintained in a nonintegrated (episomal) form, hrHPV has the ability to integrate into the host genome, ${ }^{4}$ ultimately leading to the overexpression of E6 and E7 viral genes. These genes may act to transform and immortalize cells via protein binding and inactivation of $\mathrm{p} 53$ and $\mathrm{pRB}$ tumor suppressor genes. ${ }^{5-7}$ Although hrHPV is implicated in virtually all cases of cervical carcinoma, it most often manifests as a self-limited resolving infection, with approximately $70 \%$ of cases resolved after 1 year and $90 \%$ of cases resolved after 2 years. ${ }^{8}$ Because the incidence of hrHPV is much higher than that of cervical neoplasia, testing for hrHPV has not been shown to be as cost-effective as a stand-alone primary screening test in the general population. ${ }^{9}$ Rather, as concluded from data generated by the Atypical Squamous Cells of Undetermined Significance/Low-Grade Squamous Intraepithelial Lesion Triage Study (the ALTS trial), hrHPV testing has been established as a primary triage test for cervical cytology specimens with atypical squamous cells of undetermined significance (ASC-US) results. ${ }^{10,11}$ An additional indication for hrHPV testing that has emerged more recently is as primary screening for women aged $>30$ years who have had a normal Papanicolaou (Pap) test. ${ }^{12,13}$ This is because of the finding that women in this subgroup have a lower incidence of hrHPV infection and a higher incidence of cervical dysplasia or carcinoma when HPV is present compared with patients aged $<30$ years. ${ }^{14,15}$

The Qiagen Hybrid Capture II (HC II; Qiagen, Valencia, CA) test is the most commonly used test for hrHPV and, along with the Cervista hrHPV test (Hologic, Marlborough, MA), is 1 of 2 available testing methods approved by the US Food and Drug Administration (FDA) ${ }^{16}$ Although the HC II has been approved when used with Qiagen Standard Transport Medium (STM) and ThinPrep specimens (transported in PreservCyt, both from Hologic), laboratories may use the HC II test with other liquid-based transport media (eg, SurePath; TriPath Imaging, Inc, Burlington, NC) provided appropriate inhouse validation studies have been completed. ${ }^{17}$ The HC II test has demonstrated similar performance in detecting high-grade dysplasia regardless of whether SurePath or ThinPrep specimens are used. ${ }^{18}$ The HC II test uses signal amplification chemiluminescence that provides an output in relative light units (RLUs). The patient samples are run with appropriate positive and negative controls that are used to validate results and calculate a cutoff value (CO), such that final testing output is expressed as a ratio of RLU to CO (RLU/CO). The CO is calculated by averaging the RLU values of triplicate high-risk samples (highrisk calibrators comprised of HPV type 16 [HPV 16] DNA). An RLU/CO value of $>1.0$ is deemed positive, but if the initial test falls between 1.0 and $2.5 \mathrm{RLU} / \mathrm{CO}$, the value is considered to be within an initial "equivocal" range, and per FDA labeling, the test must be repeated. If either of 2 subsequent tests fall on or above 1.0 RLU/CO, the test is then deemed positive. If 2 subsequent tests fall below 1.0, the final result is then determined to be negative for HPV.

To the best of our knowledge, few studies to date have examined the subsequent cervical histology from patients whose cervical hrHPV tests have shown "equivocal" results. ${ }^{19}$ In addition, to our knowledge only a few studies to date have examined the relation between the level of hrHPV found in cervical specimens and the incidence of cervical dysplasia detected on follow-up histology. ${ }^{20,21}$ Because the HC II is a qualitative as well as semiquantitative test, the RLU/CO value may be used as a surrogate for the hrHPV level (ie, copy number). In this study, we further examined the relation between the hrHPV level and the presence of dysplasia on follow-up histology.

\section{MATERIALS AND METHODS}

\section{Patient Sample Selection}

The study examined all hrHPV tests over an 18-month period in our laboratory, corresponding to a total volume of $5297 \mathrm{hrHPV}$ tests. During this time period, 72,056 
Table 1. Correlation of Cytologic Diagnoses With hrHPV Testing and Follow-Up Histologic Results

\begin{tabular}{|c|c|c|c|c|c|c|c|c|}
\hline \multirow{2}{*}{$\begin{array}{l}\text { Cytologic } \\
\text { Diagnosis }\end{array}$} & \multirow{2}{*}{$\begin{array}{l}\text { No. With } \\
\text { Initial } \\
\text { Equivocal } \\
\text { hrHPV Test }\end{array}$} & \multirow{2}{*}{$\begin{array}{l}\text { No. With } \\
\text { Final Positive } \\
\text { hrHPV Result }\end{array}$} & \multirow{2}{*}{$\begin{array}{l}\text { No. With } \\
\text { Follow-Up } \\
\text { Histology }\end{array}$} & \multicolumn{5}{|c|}{ Follow-Up Histologic Results } \\
\hline & & & & $\begin{array}{l}\text { Negative or } \\
\text { Reactive }\end{array}$ & CIN I & CIN II & CIN III & $\begin{array}{l}\text { Invasive } \\
\text { ADC }\end{array}$ \\
\hline NILM & 37 & 33 & 8 & $7(88 \%)$ & $1(13 \%)$ & 0 & 0 & 0 \\
\hline ASC-US & 148 & 145 & 85 & $48(56.5 \%)$ & $23(27 \%)$ & $8(9.4 \%)$ & $5(5.9 \%)$ & $1(1.2 \%)$ \\
\hline ASC-H & 13 & 13 & 11 & $4(36.4 \%)$ & $4(36.4 \%)$ & 0 & $3(27.2 \%)$ & 0 \\
\hline LSIL & 9 & 9 & 6 & $3(50 \%)$ & $3(50 \%)$ & 0 & 0 & 0 \\
\hline HSIL & 1 & 1 & 1 & $1(100 \%)$ & 0 & 0 & 0 & 0 \\
\hline AGC-US & 1 & 1 & 1 & $1(100 \%)$ & 0 & 0 & 0 & 0 \\
\hline Total & 209 & 202 & 112 & 64 (57.1\%) & 31 (27.7\%) & 8 (7.1\%) & 8 (7.1\%) & $1(0.9 \%)$ \\
\hline
\end{tabular}

hrHPV indicates high-risk human papillomavirus; CIN, cervical intraepithelial neoplasia; ADC, adenocarcinoma; NILM, negative for intraepithelial lesion or malignancy; ASC-US, atypical squamous cells of undetermined significance; ASC-H, atypical squamous cells, cannot exclude high-grade squamous intraepithelial lesion; LSIL, low-grade squamous intraepithelial lesion; HSIL, high-grade squamous intraepithelial lesion; AGC-US, atypical glandular cells of undetermined significance.

liquid-based Pap tests were completed at the Massachusetts General Hospital, of which $>97 \%$ represented SurePath specimens. hrHPV tests that were initially reported to be within the equivocal range were selected for review of subsequent hrHPV test results, including corresponding cytopathologic and histopathologic results over a 2year follow-up period. A subset of equivocal hrHPV tests corresponding to Pap tests demonstrating ASC-US cytology were reviewed and compared with a numerically matched cohort of patients who had ASC-US cytology and an associated unequivocal positive hrHPV test. This latter group was selected based on consecutive cases tested for hrHPV during the study time period ( $\mathrm{n}=148$ patients).

\section{High-Risk HPV Assay}

The HC II hrHPV Assay is an in vitro nucleic acid hybridization assay that detects 13 high-risk types of HPV (specifically, types $16,18,31,33,35,39,45,51,52,56,58$, 59 , and 68). The assay was performed as reported previously. ${ }^{19}$ Briefly, an aliquot of residual Pap test preservative medium from either a SurePath or ThinPrep Pap test was incubated with a probe cocktail containing hrHPV-specific RNA molecules, performed on 96-well plates. After hybridization, antibodies conjugated with alkaline phosphatase and specific for RNA:DNA hybrids were added, followed by a chemiluminescent substrate. Results were quantified via luminometer. Positive and negative controls were comprised of HPV-16 DNA and non-hrHPV DNA, respectively. SurePath samples were prepared for the HC II assay as previously described. ${ }^{17}$ ThinPrep samples were prepared for the HC II assay as per the manufacturer's FDA-approved protocol. Results were reported as specified for ThinPrep sample labeling, and as specified earlier.

\section{Cytopathologic and Histopathologic Preparation and Staining}

Cytopathologic and histopathologic results were based on a review of cytology and histology reports generated during the 22-month time frame of the study and extended to a period of up to 2 years after the index Pap test on which the hrHPV test was based. Histopathological results of cervical intraepithelial neoplasia (CIN) type II (CIN II) or CIN III were re-reviewed and confirmed by 2 pathologists for this study.

\section{RESULTS}

\section{Cytologic and Histologic Results of All Equivocal Tests}

The total cohort of patients whose hrHPV tests demonstrated "equivocal" results was 209 (4\%) of 5297 total tests over the 18-month period of study. These tests were found to be positive for hrHPV at the time of retesting in 202 of 209 (97\%) cases (Table 1). Of the 209 initial equivocal hrHPV tests, 204 were obtained from SurePath samples (final positive, 198 of 204 tests [97.1\%]) and 5 were obtained from ThinPrep samples (final positive, 4 of 5 tests [80\%]). Corresponding cytologic diagnoses for the 

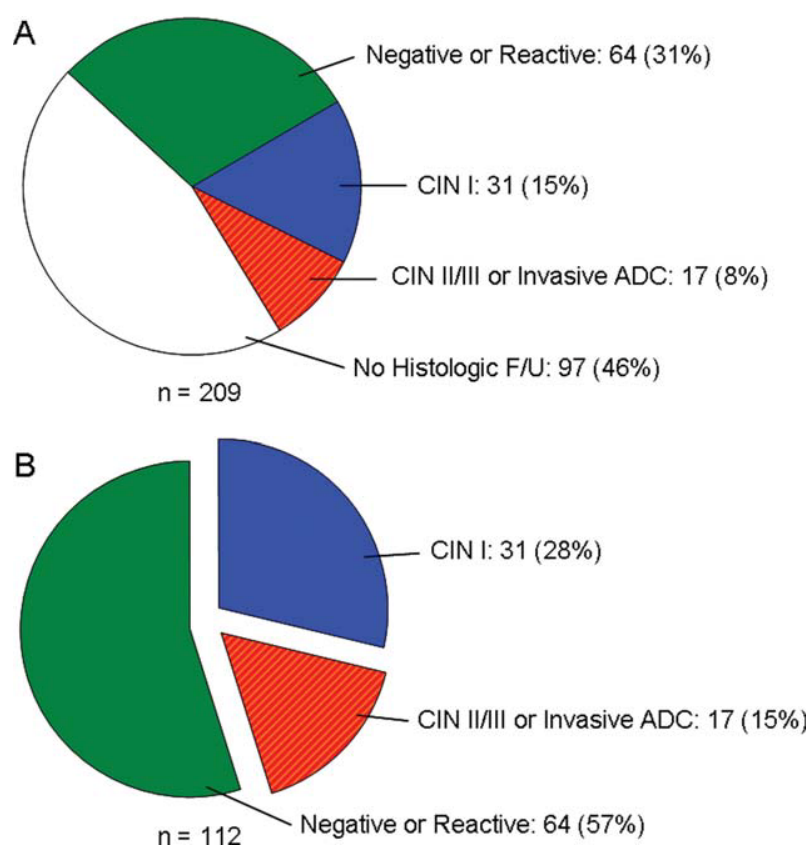

FIGURE 1. Corresponding histologic diagnoses of samples with equivocal high-risk human papillomavirus (hrHPV) tests are shown (2-year follow-up $[F / U]$ ). (A) Negative or reactive for intraepithelial lesion or malignancy and cervical intraepithelial neoplasia type I (CIN I) or CIN II-III/adenocarcinoma $(A D C)$ results are shown as percentages of the referenced total study group. (B) The results from Panel A are presented as percentages of the biopsied cohort.

209 equivocal cases (Table 1) were negative or reactive for intraepithelial lesion or malignancy (NILM) in 37 cases (18\%); ASC-US in 148 cases (71\%); atypical squamous cells, cannot exclude high-grade squamous intraepithelial lesion (ASC-H) in 13 cases (6\%); low-grade squamous intraepithelial lesion (LSIL) in 9 cases (4\%); high-grade squamous intraepithelial lesion (HSIL) in 1 case $(0.5 \%)$; and atypical glandular cells in 1 case (0.5\%). Follow-up histology was available for 112 of the 209 cases (54\%) (Table 1) (Fig. 1). Figure 1 shows data expressed as percentages of the total number of equivocal tests (NILM, $31 \%$; CIN I, $15 \%$; and CIN II/III or invasive adenocarcinoma, $8 \%)$ and percentages of the biopsied cohort (NILM, 57\%; CIN I, 28\%; CIN II/III or invasive adenocarcinoma, 15\%). Of the 7 tests that were found to be negative for hrHPV at the time of retest, 6 had no histologic follow-up and 1 demonstrated CIN I on histologic follow-up (originally ASC-US on cytology). The 5 ThinPrep specimens all demonstrated ASC-US results with no available follow-up histology.
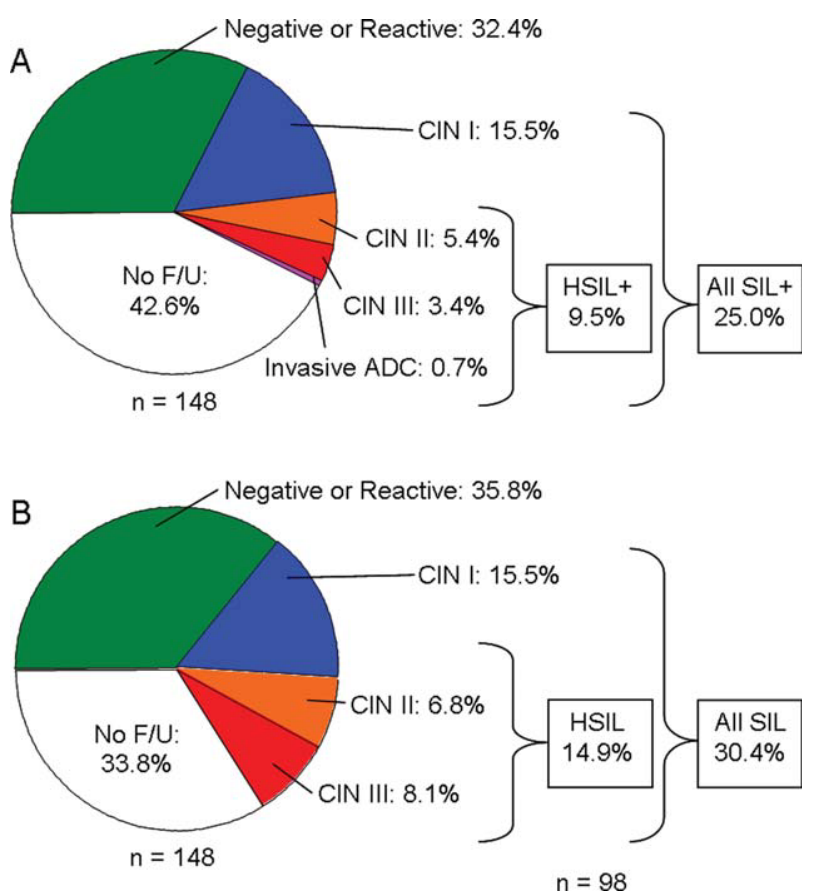

FIGURE 2. Corresponding histologic diagnoses of samples with equivocal high-risk human papillomavirus (hrHPV) tests or unequivocal positive hrHPV tests (2-year follow-up [F/U]) after a cytologic diagnosis of atypical squamous cells of undetermined significance are shown. (A) Negative or reactive for intraepithelial lesion or malignancy (NILM), cervical intraepithelial neoplasia type I (CIN I), CIN II, CIN III, and invasive adenocarcinoma (ADC) or no follow-up results are expressed as a percentage of the total cohort after an equivocal hrHPV test. (B) NILM, CIN I, CIN II, or CIN III or no follow-up results are expressed as a percentage of the total cohort after an unequivocal positive hrHPV test. HSIL indicates high-grade squamous intraepithelial lesion; +, positive; $\mathrm{SIL}$, squamous intraepithelial lesion.

\section{Histologic Results of Equivocal and Nonequivocal Tests With ASC-US Cytology}

Histologic results from the cohort of patients with an equivocal hrHPV test demonstrating ASC-US on the corresponding Pap test were compared with histologic results from a numerically matched cohort of patients with a unequivocal hrHPV test and ASC-US demonstrated on a corresponding Pap test (Figs. 2 and 3). Figure 2 shows data expressed as percentages of the total number of hrHPV tests. For patients with an equivocal hrHPV test, follow-up biopsy data indicated NILM in $32.4 \%$, CIN I in $15.5 \%$, and CIN II/III or invasive adenocarcinoma in $9.5 \%$. For patients with an unequivocal positive hrHPV test, follow-up biopsy data demonstrated NILM in $35.8 \%$, CIN I in $15.5 \%$, and CIN II/III in $14.9 \%$. Figure 

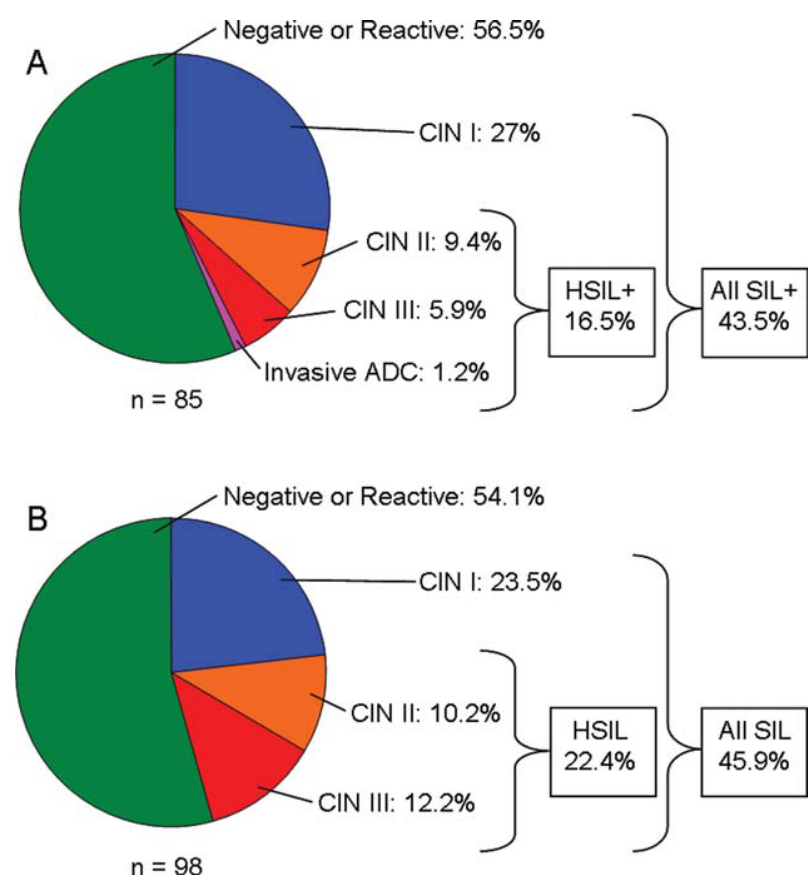

FIGURE 3. Corresponding histologic diagnoses of samples with equivocal high-risk human papillomavirus (hrHPV) tests or unequivocal positive hrHPV tests (2-year follow-up) after a cytologic diagnosis of atypical squamous cells of undetermined significance are shown. (A) Negative or reactive for intraepithelial lesion or malignancy (NILM), cervical intraepithelial neoplasia type I (CIN I), CIN II, CIN III, or invasive adenocarcinoma (ADC) results are expressed as a percentage of the biopsied cohort after an equivocal hrHPV test. (B) NILM, CIN I, CIN II, or CIN III results are expressed as a percentage of the biopsied cohort after an unequivocal positive hrHPV test. HSIL indicates high-grade squamous intraepithelial lesion; +, positive; SIL, squamous intraepithelial lesion.

3 shows data expressed as percentages of the biopsied cohort. For patients with an equivocal hrHPV test, follow-up biopsy data demonstrated NILM in 56.5\%, CIN I in $27 \%$, and CIN II/III or invasive adenocarcinoma in $16.5 \%$. For patients with an unequivocal positive hrHPV test, follow-up biopsy data indicated NILM in 35.8\%, CIN I in $15.5 \%$, and CIN II/III in $14.9 \%$.

\section{Chemiluminescence Intensity of hrHPV Tests Based on Histologic Diagnosis}

The chemiluminescence intensity of unequivocal positive hrHPV tests from patients found to have ASC-US cytology on a corresponding Pap test was correlated with the follow-up histologic results (Fig. 4). Average chemiluminescence intensity (shown as RLU/CO) when correlated with follow-up histology was 281 in negative cases, 153 in

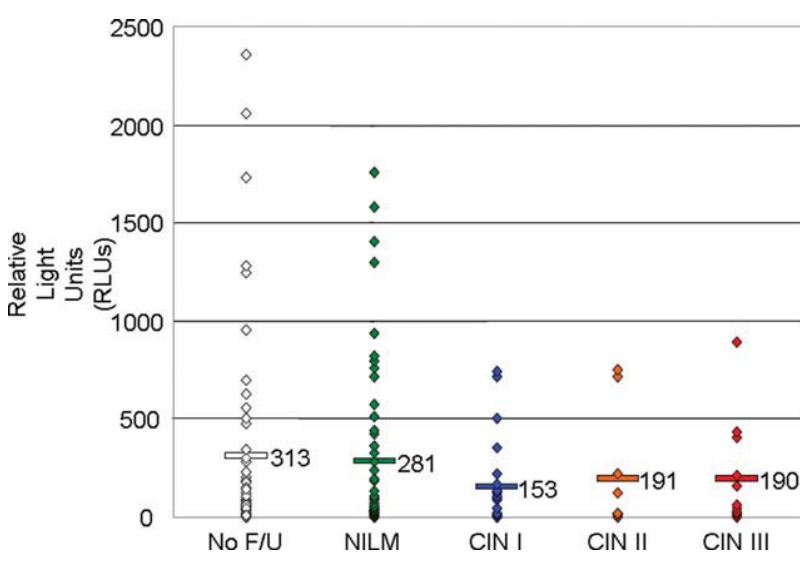

FIGURE 4. Corresponding relative light units (RLU)/cutoff value (CO) ratios of high-risk human papillomavirus tests from samples demonstrating atypical squamous cells of undetermined significance on cervical cytology and correlated with follow-up cervical biopsies determined to be negative or reactive for intraepithelial lesion or malignancy (NILM), cervical intraepithelial neoplasia type I (CIN I), CIN II, and CIN III are shown.

CIN I cases, 191 in CIN II cases, 190 in CIN III cases, and 313 in no follow-up cases. The differences among the groups were not found to be statistically significant (independent group Student $t$ test). The calculated 2-tailed $P$ values were CIN II or CIN III versus CIN I, $P=.61$; CIN II or CIN III versus NILM, $P=.37$; and CIN I versus NILM, $P=.19$.

\section{Histologic Results Based on hrHPV Test Chemiluminescence Intensity}

Follow-up histology corresponding to patients found to have ASC-US on cytology and a concomitant nonequivocal positive hrHPV test were correlated with chemiluminescence intensity. The median hrHPV chemiluminescence intensity of 148 cases was 61.54 RLU/CO. Figure 5 shows the resulting follow-up histology of patients corresponding to those whose RLU/CO value was below the median (range, 2.74-57.71 RLU/CO) or above the median (range, 65.36-2361.88 RLU/CO). The follow-up histology of samples corresponding to hrHPV tests with chemiluminescence intensity that was below the median was no follow-up in $29.7 \%$ of samples, negative for dysplasia in $37.8 \%$ of samples, CIN I in $16.2 \%$ of samples, or CIN II/III in $16.2 \%$ of samples. The followup histology of samples corresponding to hrHPV tests with chemiluminescence intensity that was above the 

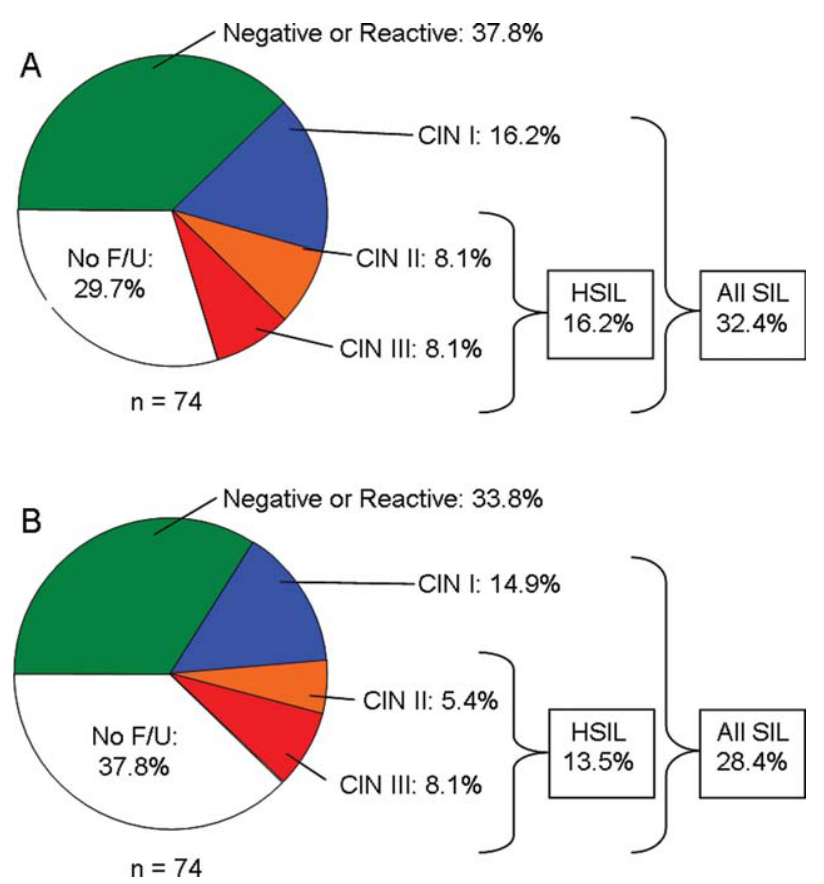

FIGURE 5. Corresponding histologic diagnoses of samples with unequivocal positive high-risk human papillomavirus (hrHPV) tests (2-year follow-up [F/U]) after a cytologic diagnosis of atypical squamous cells of undetermined significance were divided into cases falling above or below the median chemiluminescence intensity. (A) Negative or reactive for intraepithelial lesion or malignancy (NILM), cervical intraepithelial neoplasia type I (CIN I), CIN II, or CIN III results were expressed as the percentage of the biopsied cohort after hrHPV test indicating chemiluminescence intensity below the median. (B) NILM, cervical intraepithelial neoplasia type I (CIN I), CIN II, or CIN III results were expressed as the percentage of the biopsied cohort after hrHPV test indicating chemiluminescence intensity above the median.

median was no follow-up in $37.8 \%$ of samples, negative for displasia in $33.8 \%$ of samples, CIN I in $14.9 \%$ of samples, or CIN II/III in $13.5 \%$ of samples. Figure 6 shows the same 2 groups expressed as a percentage of the biopsied cohort. The available follow-up histology of samples corresponding to hrHPV tests with RLU/CO values $>1000$ ( $\mathrm{n}=19$ samples) were NILM in 7 samples $(37 \%)$, CIN I in 7 samples (37\%), or CIN II/III in 5 samples (26\%). Figure 7 shows the histologic follow-up when the groups were divided into 4 equal subsets based on chemiluminescence intensity.

\section{DISCUSSION}

The main objectives of the current study were 1) to provide 2-year histologic follow-up for the assessment of pa-
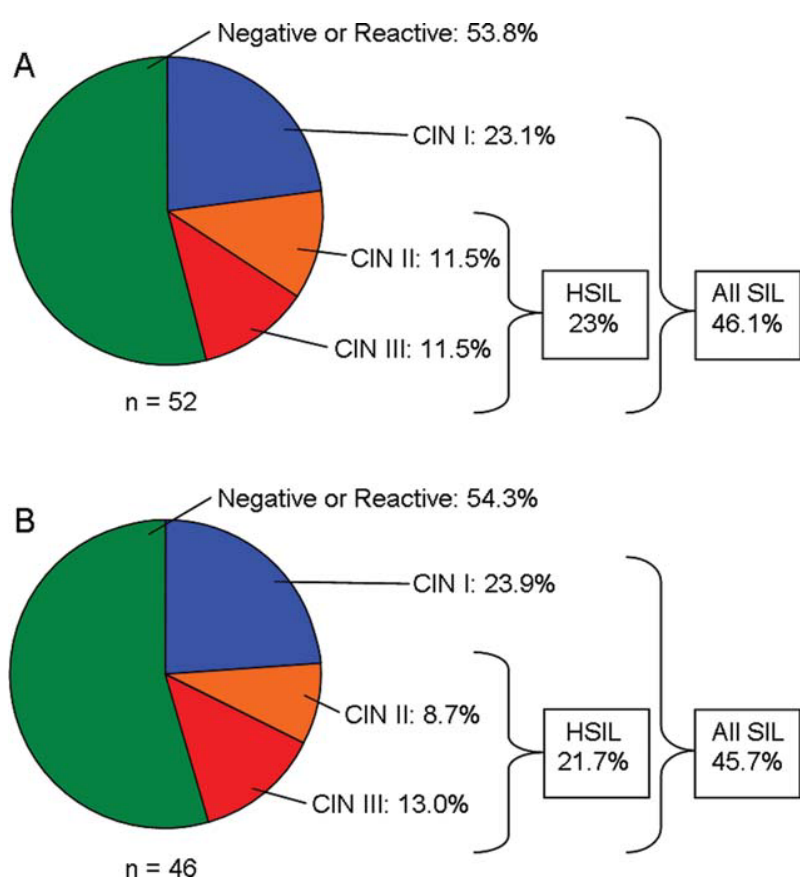

FIGURE 6. Corresponding histologic diagnoses of samples with unequivocal positive high-risk human papillomavirus (hrHPV tests) (2-year follow-up [F/U]) after a cytologic diagnosis of atypical squamous cells of undetermined significance were divided into cases falling above or below the median chemiluminescence intensity. (A) Negative or reactive for intraepithelial lesion or malignancy (NILM), cervical intraepithelial neoplasia type I (CIN I), CIN II, or CIN III results were expressed as the percentage of the biopsied cohort after an hrHPV test indicating chemiluminescence below the median. (B) NILM, CIN I, CIN II, or CIN III results were expressed as the percentage of the biopsied cohort after an hrHPV test indicated chemiluminescence above the median. HSIL indicates high-grade squamous intraepithelial lesion; SIL, squamous intraepithelial lesion.

thology associated with equivocal hrHPV results; 2) compare follow-up dysplasia rates after ASC-US Pap test results and equivocal hrHPV tests with dysplasia rates in a similar group of patients with unequivocal positive hrHPV results; and 3) correlate the chemiluminescent intensity of a positive HC II hrHPV test with the risk of cervical dysplasia on a follow-up cervical biopsy. We previously reported a $4 \%$ initial equivocal hrHPV test rate and the subsequent conversion of $97.4 \%$ of those tests to positive at the time of retesting. ${ }^{19}$ This number is higher than that presented in the manufacturer's packaging insert when examining the final hrHPV positive rate after testing equivocal samples. The manufacturer found a subsequent final positive rate of 177 of 219 samples (81\%) when using PreservCyt as the specimen medium and 127 


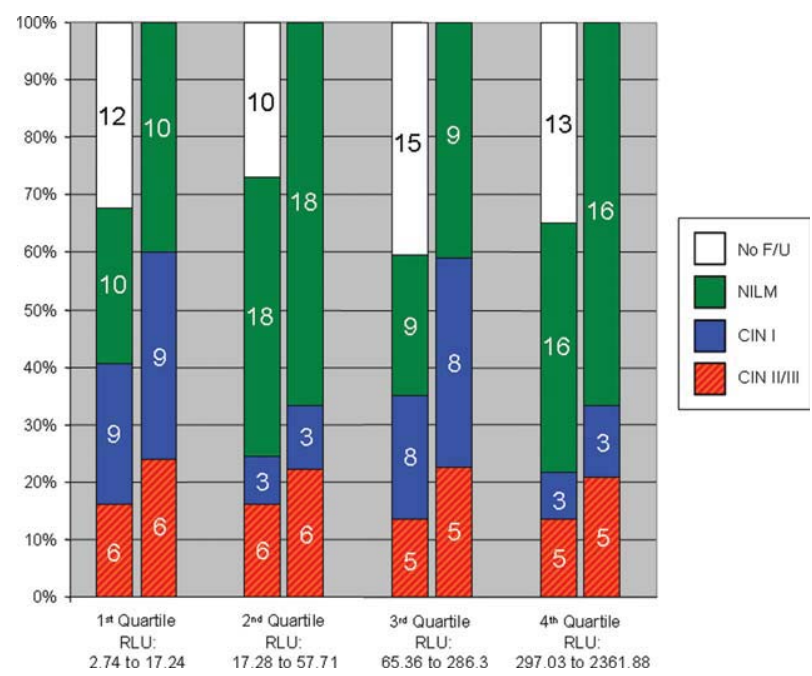

FIGURE 7. Corresponding relative light units (RLU)/cutoff value (CO) ratios of high-risk human papillomavirus tests from samples demonstrating atypical cells of undetermined significance on cervical cytology and correlated with followup cervical biopsies determined to be negative or reactive for intraepithelial lesion or malignancy (NILM), cervical intraepithelial neoplasia type I (CIN I), CIN II, and CIN III are shown. The left bar in each pair corresponds to all cases $(n=37$ for each group), whereas the right bar corresponds to the biopsied cohort.

of 140 samples (91\%) when using STM as the specimen medium. On the basis of these data alone, and not followup histology, the manufacturer recommends repeat testing when specimens are preserved in PreservCyt but does not recommend retesting when specimens are preserved in STM due to the higher reproducibility of the latter. Given that our retesting results demonstrated higher reproducibility (ie, 97\%) than either of the manufacturer's tests, this lends strong support to the conclusion that these tests do not need to be repeated. The current study is comprised of samples preserved predominantly in SurePath media, which has been shown to exhibit reproducible results with the HC II hrHPV assay at the time of retesting. ${ }^{17,22}$ SurePath is the predominant liquid-based media (>97\%) used for Pap tests at the reference institution, and therefore the preponderance of SurePath media in the current study reflects the mix of Pap tests at the reference institution and not inherent qualities of either SurePath or ThinPrep media.

The best rationale for treating these "equivocal" tests as true-positive results can be found in examining the follow-up histologic results. Thus, in addition to retesting, we explored the 2-year histologic follow-up of cervical biopsies associated with the "equivocal" tests to definitively determine whether these tests differ with regard to unequivocal positive hrHPV tests. Because dysplasia rates may vary depending on the patient population being served, ${ }^{10}$ we compared follow-up histology from "equivocal" hrHPV tests with unequivocal positive hrHPV tests in our laboratory. In our laboratory, the 2-year total risk of dysplasia (CIN I/II/III) after an ASC-US Pap test was $43.5 \%$ or $45.9 \%$ of the biopsied cohort for "equivocal" or positive hrHPV tests, respectively; and the 2-year risk of high-grade dysplasia (CIN II/III) after an ASC-US Pap test was $16.5 \%$ or $22.4 \%$ of the biopsied cohort for "equivocal" or positive hrHPV tests, respectively. As a comparison, the ALTS trial reported a 2-year follow-up rate of high-grade dysplasia of $26 \%$ in their biopsied cohort, which was an average of the rates found among the 4 participating institutions. ${ }^{10,11}$ Thus, on the basis of the outcome of either hrHPV retesting or subsequent histologic biopsy, data from the current study suggest that hrHPV tests with an initial value $>1.0$ RLU/CO be treated as an unequivocal positive hrHPV test, and that repeat testing of these samples is unnecessary.

To our knowledge, few studies to date have examined the significance of "equivocal" hrHPV results obtained from HC II. ${ }^{23}$ To our knowledge, the current study is the first to provide a broad picture of the predictive value of chemiluminescence intensity derived from the HC II hrHPV test from the lowest, most equivocally positive specimens to the highest values generally obtained in laboratories. Although to the best of our knowledge no other single institution has examined this on as broad a scale, several other studies have examined the relation between hrHPV viral load and subsequently detected cervical dysplasia. ${ }^{24-29}$ The recently published series from Sargent et $\mathrm{al}^{29}$ reported a reduced rate of high-grade dysplasia after an hrHPV test, with an RLU/CO value of between 1 and 2 (ie, 9.2\% vs $17.9 \%$ for an RLU/CO value $\geq 2.0$ ), although their methodology differs considerably from that of the current present study (eg, combining "borderline" and "mild" cytology). Nevertheless, the indicated rate of $9.2 \%$ represents a nearly 5 -fold risk compared with that associated with negative hrHPV tests in the study by Sargent et al. ${ }^{29}$ Using polymerase chain reaction-based hrHPV detection, an increase in the viral load of carcinogenic subtypes demonstrates a positive correlation with cervical abnormalities in the general population 
(ie, when all Pap diagnoses are included), ${ }^{26}$ and the association between type-specific HPV-16 viral load with CIN III or cancer indicates a positive correlation in patients with negative cervical cytology, but not those with ASCUS cytology. ${ }^{24}$ When HC II was used to detect hrHPV subtypes, no association was noted between increasing viral load and the risk of CIN III or cancer, ${ }^{27}$ although a modest positive correlation was observed for CIN I. ${ }^{21}$ Further analysis of specimens gathered from the ALTS trial demonstrated that higher hrHPV viral load is associated with a greater prevalence of multiple hrHPV subtypes and exfoliated ASC-US/LSIL cells, but not exfoliated ASC-H or HSIL cells. ${ }^{28}$ Thus, these studies suggest that using hrHPV viral load to predict severe dysplasia in a clinically relevant setting is not possible. Data from the current study lend further support to this hypothesis, because we examined a highly clinically relevant population (ie, a population with hrHPV positivity and ASC-US cytology) and found no correlation with viral load and the presence or severity of subsequently detected dysplasia. We chose to divide patients evenly into either halves or quartiles based on HC II chemiluminescence intensity and found no significant difference between either low-grade or high-grade dysplasia. Other methods of segregating samples based on viral load (eg, an RLU/CO value $>1000$ ) also failed to demonstrate a correlation among the current study data, although our dataset for specimens with a RLU/CO value $>1000$ was small because such specimens comprise a small subset of positive hrHPV tests (ie, 6\% in the current series). In addition, this subset has been examined exclusively in another study and has been shown to be associated with a modest increase of low-grade (CIN I) lesions, but not high-grade lesions (ie, CIN II or above). ${ }^{21}$ Presumably, low-grade dysplasias may demonstrate a higher viral load given that these infections are productive, nonintegrated infections, and active viral replication is taking place. The results of the current study cannot fully address this theory because we were unable to ascertain the presence of absence of CIN I in those cases in which a CIN II or CIN III lesion was detected; thus, CIN I lesions may be found in any or all cases demonstrating CIN II or CIN III. The histologic results of each biopsy were reported based on the highest grade of dysplasia present as opposed to all grades of dysplasia that were present. In addition, colposcopically directed biopsies are aimed at sampling the highest grades of dysplasia versus detecting all areas of low-grade dysplasia that may be present. Thus, the complex and most likely multiple opposing sets of biologic principals affecting the association between viral load and dysplasia are difficult to dissect in the current study. However, the relevant association between viral load and high-grade dysplasia in clinically relevant samples is not difficult to dissect: the results of the current study strongly refute the belief that viral load can predict the degree or presence of high-grade dysplasia in histologic cervical samples after an abnormal Pap test.

\section{CONFLICT OF INTEREST DISCLOSURES}

The authors made no disclosures.

\section{REFERENCES}

1. Walboomers JM, Jacobs MV, Manos MM, et al. Human papillomavirus is a necessary cause of invasive cervical cancer worldwide. J Pathol. 1999;189:12-19.

2. Clavel C, Masure M, Bory JP, et al. Human papillomavirus testing in primary screening for the detection of high-grade cervical lesions: a study of 7932 women. Br J Cancer. 2001;84:1616-1623.

3. Stoler MH, Castle PE, Solomon D, Schiffman M. The expanded use of HPV testing in gynecologic practice per ASCCP-guided management requires the use of well-validated assays. Am J Clin Pathol. 2007;127:335-337.

4. zur Hausen H. Papillomaviruses and cancer: from basic studies to clinical application. Nat Rev Cancer. 2002;2:342350.

5. Munger K. Disruption of oncogene/tumor suppressor networks during human carcinogenesis. Cancer Invest. 2002;20:71-81.

6. Riley RR, Duensing S, Brake T, Munger K, Lambert PF, Arbeit JM. Dissection of human papillomavirus E6 and E7 function in transgenic mouse models of cervical carcinogenesis. Cancer Res. 2003;63:4862-4871.

7. zur Hausen H. Papillomaviruses causing cancer: evasion from host-cell control in early events in carcinogenesis. J Natl Cancer Inst. 2000;92:690-698.

8. Castle PE, Sideri M, Jeronimo J, Solomon D, Schiffman M. Risk assessment to guide the prevention of cervical cancer. Am J Obstet Gynecol. 2007;197:356 e1-6.

9. Goldie SJ, Kim JJ, Myers E. Chapter 19: Cost-effectiveness of cervical cancer screening. Vaccine. 2006;24(suppl 3):S3/ 164-170.

10. Solomon D, Schiffman M, Tarone R. Comparison of 3 management strategies for patients with atypical squamous cells of undetermined significance: baseline results from a randomized trial. J Natl Cancer Inst. 2001;93:293-299. 
11. Solomon D, Schiffman M, Tarone R. ASCUS LSIL Triage Study (ALTS) conclusions reaffirmed: response to a November 2001 commentary. Obstet Gynecol. 2002;99:671674.

12. Goldie SJ, Kim JJ, Wright TC. Cost-effectiveness of human papillomavirus DNA testing for cervical cancer screening in women aged 30 years or more. Obstet Gynecol. 2004;103: 619-631.

13. Wright TC Jr, Massad LS, Dunton CJ, Spitzer M, Wilkinson EJ, Solomon D. 2006 consensus guidelines for the management of women with cervical intraepithelial neoplasia or adenocarcinoma in situ. Am J Obstet Gynecol. 2007;197:340-345.

14. Cuzick J, Clavel C, Petry KU, et al. Overview of the European and North American studies on HPV testing in primary cervical cancer screening. Int J Cancer. 2006;119: 1095-1101.

15. Dillner J, Rebolj M, Birembaut P, et al. Long term predictive values of cytology and human papillomavirus testing in cervical cancer screening: joint European cohort study. BMJ. 2008;337:a1754.

16. Kurtycz DF, Smith M, He R, Miyazaki K, Shalkham J. Comparison of methods trial for high-risk HPV. Diagn Cytopathol. 2010;38:104-108.

17. Ko V, Tambouret RH, Kuebler DL, Black-Schaffer WS, Wilbur DC. Human papillomavirus testing using hybrid capture II with SurePath collection: initial evaluation and longitudinal data provide clinical validation for this method. Cancer (Cancer Cytopathol). 2006;108:468-474.

18. Siddiqi A, Spataro M, McIntire H, et al. Hybrid capture 2 human papillomavirus DNA testing for women with atypical squamous cells of undetermined significance papanicolaou results in SurePath and ThinPrep specimens. Cancer (Cancer Cytopathol). 2009;117:318-325.

19. Knoepp SM, Kuebler DL, Wilbur DC. Resolution of equivocal results with the Hybrid Capture II high-risk HPV DNA test: a cytologic/histologic review of 191 cases. Diagn Mol Pathol. 2007;16:125-129.

20. Tsai HT, Wu CH, Lai HL, et al. Association between quantitative high-risk human papillomavirus DNA load and cervical intraepithelial neoplasm risk. Cancer Epidemiol Biomarkers Prev. 2005;14(11 pt 1):2544-2549.

21. Yuan Q, Wilbur DC. Original cervical cytology and follow-up biopsy results in positive high risk human papillomavirus DNA tests with high-level results. Acta Cytol. 2008;52:557-562.

22. Kuebler DL, Illingworth A, Blenc AM, Wilbur DC. A peer comparison program for the quality assurance of human papillomavirus DNA detection using the Digene Hybrid Capture II/SurePath method shows excellent analytic interlaboratory correlation. Cancer. 2007;111:339-343.

23. Federschneider JM, Yuan L, Brodsky J, Breslin G, Betensky RA, Crum CP. The borderline or weakly positive Hybrid Capture II HPV test: a statistical and comparative (PCR) analysis. Am J Obstet Gynecol. 2004;191:757-761.

24. Castle PE, Schiffman M, Scott DR, et al. Semiquantitative human papillomavirus type 16 viral load and the prospective risk of cervical precancer and cancer. Cancer Epidemiol Biomarkers Prev. 2005;14:1311-1314.

25. Jarboe EA, Venkat P, Hirsch MS, Cibas ES, Crum CP, Garner EO. A weakly positive HPV Hybrid Capture II result correlates with a significantly lower risk of HSIL following ASC-US cytology. Mod Pathol. 2008;21:207a208a.

26. Kovacic MB, Castle PE, Herrero R, et al. Relationships of human papillomavirus type, qualitative viral load, and age with cytologic abnormality. Cancer Res. 2006;66:1011210119.

27. Lorincz AT, Castle PE, Sherman ME, et al. Viral load of human papillomavirus and risk of CIN3 or cervical cancer. Lancet. 2002;360:228-229.

28. Sherman ME, Wang SS, Wheeler CM, et al. Determinants of human papillomavirus load among women with histological cervical intraepithelial neoplasia 3: dominant impact of surrounding low-grade lesions. Cancer Epidemiol Biomarkers Prev. 2003;12:1038-1044.

29. Sargent A, Bailey A, Turner A, et al. Optimal threshold for a positive hybrid capture 2 test for detection of human papillomavirus: data from the ARTISTIC trial. I Clin Microbiol. 2010;48:554-558. 